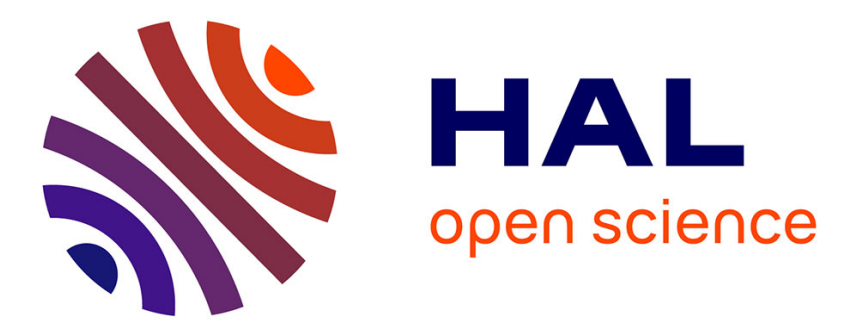

\title{
Enhanced Clustering Approach for Traffic Regulation in Directional Antennas based Nanonetworks
}

\author{
Lina Aliouat, Hakim Mabed, Julien Bourgeois
}

\section{To cite this version:}

Lina Aliouat, Hakim Mabed, Julien Bourgeois. Enhanced Clustering Approach for Traffic Regulation in Directional Antennas based Nanonetworks. Annual Symposium on Applied Computing, Mar 2020, Brno, Czech Republic. hal-02993850

\section{HAL Id: hal-02993850 \\ https://hal.science/hal-02993850}

Submitted on 7 Nov 2020

HAL is a multi-disciplinary open access archive for the deposit and dissemination of scientific research documents, whether they are published or not. The documents may come from teaching and research institutions in France or abroad, or from public or private research centers.
L'archive ouverte pluridisciplinaire HAL, est destinée au dépôt et à la diffusion de documents scientifiques de niveau recherche, publiés ou non, émanant des établissements d'enseignement et de recherche français ou étrangers, des laboratoires publics ou privés. 


\section{Enhanced Clustering Approach for Traffic Regulation in Directional Antennas based Nanonetworks}

\author{
Lina Aliouat \\ lina.aliouat@univ-fcomte.fr \\ Univ. Bourgogne Franche-Comté \\ Montbéliard, France
}

\author{
Hakim Mabed \\ hmabed@femto-st.fr \\ Univ. Bourgogne Franche-Comté \\ Montbéliard, France
}

\author{
Julien Bourgeois \\ julien.bourgeois@femto-st.fr \\ Univ. Bourgogne Franche-Comté \\ Montbéliard, France
}

\begin{abstract}
Wireless NanoNetworks (WNN) are gaining an increasing interest and attracting many researchers from the networking community. This is due to the huge potential offered by nanodevices development. A WNN is formed by a high number of nanonodes utilizing specific properties of nanomaterials and nanoparticles to act at nanometer scale. However, the intrinsic limitations of nanonodes (energy, memory, communication range, and computation capacity), impose WNN protocols to be adapted to these stringent requirements.

Furthermore, the extremely high density of nanonodes leads to congestion phenomena, requiring thorough traffic regulation mechanisms in order to reduce hindering radio signal collisions. Traffic regulation aims also to reduce valuable energy consumption. In this paper, we operate with Terahertz directional antennas in order to efficiently direct the radio signals towards the targeted area which significantly reduces interference. For regulating the exchanged messages, we propose a synchronous communication protocol based on a clustering approach where intra and inter cluster communications will be achieved respectively by a Time Division Multiple Access (TDMA) protocol, and a new distributed TDMA combined with Forwarding Dominate Nodes (FDN) protocol.

Several scenarios have been carried out and the obtained simulation results show that our proposition significantly reduces both the number of collisions and the number of exchanged messages required to broadcast data from the nanonodes.
\end{abstract}

\section{KEYWORDS}

Nanonetworks, Directional Antennas, Clustering, Distributed TDMA, Traffic Regulation.

\section{ACM Reference Format:}

Lina Aliouat, Hakim Mabed, and Julien Bourgeois. 2020. Enhanced Clustering Approach for Traffic Regulation in Directional Antennas based Nanonetworks. In The 35th ACM/SIGAPP Symposium on Applied Computing (SAC '20), March 30-April 3, 2020, Brno, Czech Republic. ACM, Brno, Czech Republic, 8 pages. https://doi.org/10.1145/3341105.3373889

Permission to make digital or hard copies of all or part of this work for personal or classroom use is granted without fee provided that copies are not made or distributed for profit or commercial advantage and that copies bear this notice and the full citation on the first page. Copyrights for components of this work owned by others than the author(s) must be honored. Abstracting with credit is permitted. To copy otherwise, or republish, to post on servers or to redistribute to lists, requires prior specific permission and/or a fee. Request permissions from permissions@acm.org.

SAC '20, March 30-April 3, 2020, Brno, Czech Republic

(C) 2020 Copyright held by the owner/author(s). Publication rights licensed to ACM.

ACM ISBN 978-1-4503-6866-7/20/03 ..\$15.00

https://doi.org/10.1145/3341105.3373889

\section{INTRODUCTION}

Over the last decade, Wireless NanoNetworks (WNN), such as Nano Sensors Networks (WNSN) [15], Body Area Network (BAN) [4] or swarm nanorobot networks [5], has received an increasing attention from the research community. This intense research activity is due to the large interest raised by wireless nanocomponents in finding out appropriate solutions to critical problems in various strategic areas. These solutions are related to connecting processor cores, sensing nanoscale events occurring in targeted environment or coordinating the actions of numerous nanodevices in order to obtain a given system behavior.

Although this technology is destined to a promising future, several obstacles have to be overcome before a real deployment [19]. These barriers are mainly due to a lack of mastery of nano objects technologies: processors, antennas, or energy generator [12] which imposes the use of new physical, chemical or biological materials [2]. In this context, graphene [7] is seen as one of the promising technology for manufacturing sub-millimeter antennas working in the Terahertz frequency band.

Besides, nanocommunication technologies open the way to the conception of systems where a high number of nodes are concentrated in a tiny space. Therefore, the traditional solutions used in standard wireless networks are not directly applicable but need to be adapted to better comply with.

\section{CONTRIBUTION}

This work is devoted to the Medium Access Control (MAC) layer for ultra dense Terahertz nanonetworks. More specifically, network nodes are organized into clusters [17] to regulate the traffic messages in order to save nodes energy and to reduce generated interference. Each cluster includes a set of nodes (members) and a Cluster Head $(\mathrm{CH})$. A CH is an ordinary node responsible for scheduling the data transmission of its members. Data received from member nodes are multiplexed and sent to the neighbor cluster heads. This scheme of accessing channel is the well known Time Division Multi-Access (TDMA) [23].

The access control is made by the $\mathrm{CHs}$. Each $\mathrm{CH}$ allocates a specific time slot to each node member of the cluster. Only this node will be able to communicate during this time slot. Therefore, intra-cluster collisions are avoided leading to less re-transmissions and better energy consumption and higher throughput. Indeed, in random access protocols and CDMA/CA protocols the number (not the size) of communications increases the probability of collisions making time scheduling-based approaches benefit.

The clustering approach is widely studied in the field of ad-hoc wireless networks $[10,21]$. However, Terahertz nanonetwork has specific features due to Terahertz signal characteristics including 
propagation phenomena such as molecular absorption [13], a very large bandwidth and various communication ranges [26]. We then present how the Terahertz band can be better managed, by varying the used frequency sub-bands within each cluster.

In addition, we consider the case of ultra dense WNN (up to thousand of neighbors per node). Major works on clustering approaches ignore the antenna steering. In our approach, we suggest the use of directional transmission/reception antennas as a mean to regulate and reduce collisions resulting from concurrent accesses on communication channels. We propose a distributed TDMA for inter-cluster communications. Finally, with the concept of the Optimized Link State Routing Protocol (OLSR) [1] we propose the Forwarding Dominating Nodes (FDN) to optimize the inter-cluster communications.

The rest of the paper is organized as follows: Section III presents related works. Section IV is devoted to our proposal adapting the clustering approach enhanced with both a new distributed TDMA protocol and FDN scheme. Section V discusses the performance evaluation of our solution via simulation. Finally, we conclude our work and present future perspectives in Section VI.

\section{RELATED WORKS}

The very high node density in wireless nanonetworks significantly affects the traffic messages regularity. Traffic coordination between nodes leads to less disruptions in the network and, as a consequence, less energy dissipation and improves the nodes longevity.

Rikhtegar et al. [22] proposed EEWNSN-MAC: Energy Efficient Wireless Nano Sensor Network MAC Protocol for mobile multihop nano wireless networks. This protocol is based on clustering nodes organization in order to reduce the effects of mobility and collisions resulting from channel accesses. The nano nodes send their data packets to nano routers (Cluster Heads) according to the TDMA strategy. The nano routers aggregate the received data and transmit it to the nano-micro interface. The latter sends it to the remote server by using a IEEE 802.11 wireless connection. The work is primarily focused on energy efficiency and communication reliability to minimize packet losses. If intra-cluster communications are done according to the classical TDMA scheduling process, the authors do not specify how inter-clusters communications are coordinated to avoid collisions.

In [20], the authors have proposed an inter-layers hierarchical protocol using three types of nodes: nano nodes that constitute the standard nodes, the nano routers similar to cluster heads and nano interface nodes. The protocol uses two routing policies, namely: selective flooding and random routing. In random routing protocol, a data packet is sent towards a nano router if possible, otherwise towards a neighboring nano node. The access to the channel is done according to two asynchronous strategies: Transparent-Mac where packets are routed from the network layer to the physical one and the Smart-Mac strategy where any packet received from the upper layer is buffered until delivery to the physical layer. Before sending out a packet, the MAC layer determines first the neighboring nodes of the sender. If such nodes exist, the packet is sent to the physical interface that uses the protocol Time Spread On-Off Keying (TSOOK) [18]. On the contrary, the sender waits a random amount of time and then repeats the discovery process of neighbors. This protocol suffers from a lack of determinism, since the coordination between the three used layers can fail if no immediate neighboring node is present. In addition, the concurrent channel accesses in a highly dense environment, like WNSN, without a plausible messages traffic control (Via collisions limitation) do not prevent occurrence of costly communication disturbances.

In [24], the authors have proposed a hierarchical MAC protocol intended for WNSNs. They consider three types of nodes: member nodes which form a cluster, a cluster head that coordinates intracluster communications and aggregates data sensed by nodes and a base station that receives aggregated data sent by cluster heads. To transmit their packets, member nodes first send authorization requests. These requests are processed by the cluster head which schedule the channel according to the strategy of First come - First served. However, the authors do not indicate how communications between clusters are carried out for reaching the base station.

The authors in [14] have proposed PHLAME: Physical Layer aware MAC protocol for Electromagnetic nanonetworks in the Terahertz band. This protocol is implemented on top of a new pulse-based communication protocol named Rate Division Time Spread On-Off Keying (RD TS-OOK). The latter is an improved version of TS-OOK which uses a coding approach that reduce the number of ' 1 ' symbols, and where the time between the consecutive symbols (symbol rate) is randomly selected for each communication. The use of different symbol rates reduces the selective interference effect where two communications using the same symbol rate are received in synchronized way by a given node (the symbols are received in the same time).

In [26], the authors have proposed an energy and spectrumaware MAC protocol which is a hierarchical protocol where all nano sensors can directly communicate with the nano routers in a single hop. Such an organization requires a certain thoroughness in the deployment and disposition of nano nodes which may therefore exclude a random deployment. This protocol aims to achieve a fair throughput and to ensure an optimal life time to channel access with an optimal harvesting and energy consumption by nano nodes. To do this, they consider a parameter named report of critical packet transmission which represents the maximum ratio allowed between the transmission duration and the amount of energy harvesting such that this collected quantity of energy is greater than the one consumed. This enables a perpetual data transmission without energy depleting. The authors admit that a nano node is capable to recharge its battery during transmission periods and sleeping state. The authors use an algorithm to schedule symbols compression applied to TS-OOK by which several nano sensors can transmit in parallel without generating collisions. However, the huge number of sensed data, issued by an ultra dense nanonetwork, is not subject to an aggregation process, this raises the costly problem of redundant transmission.

\section{TRAFFIC REGULATION FOR TERAHERTZ NANONETWORKS}

Figure 1 shows that the proposed protocol operates with rounds $T R$. Each round is triggered in synchronious way over all nodes using a specific message sent by the base station. A round is composed of two phases. The clustering phase performs the $\mathrm{CH}$ selection, 
$A D V$, FOIN, FDN and intra/inter TDMA. The second phase ensures data transmission. In order to reduce collisions, each node uses its directional antenna to communicate with the others. For this the following section describes the directional antennas configuration that takes place before the two precedent phases.

\subsection{Directional Antennas}

The use of beam steerable antennas are vital for Terahertz nanonetworks applications due to the high path loss, signal attenuation and the energy consumption constraints. In the presence of dense nanonetwork, beam steerable antennas allow to reduce the interference by concentrating radio signal on the targeted area. The beam steering techniques could be classified into three main categories [25]: mechanical steering, beamforming and reflectarray antenna. Mechanical steering [6] is considered unsuitable regarding its impact of antenna size and its lack of responsiveness. Beamforming techniques [16] use a combination of signals provided by an array of antenna leading to highly directional radio signal. Reflectarray antenna [8] techniques use an array antenna combined with a reflector. The signal provided by a field generator is reflected by a set of reflectors in a specific direction.

Consequently, in this work we assume that each node has a reconfigurable directional antenna. The latter can be dynamically directed to cover a particular area using $D$ specific configurations. A node changes the parameters of its antenna in a cyclic manner and at a regular interval of time among a set of $D$ possible directions.

At the beginning, the synchronization station sends a message to start the clustering phase. At the end of this phase, the nanonetwork is subdivided into clusters, each one contains: a $\mathrm{CH}$ node and cluster member nodes, named ordinary nodes. The duration of a complete cycle $T C$, i.e. the time to cover all possible directions, depends on the node type ( $\mathrm{CH}$ or member node). The reconfiguration frequency of $\mathrm{CH}$ node is $\mathrm{D}$ times slower than reconfiguration frequency of ordinary nodes.

$$
T C_{C H}=T C_{\text {Ordinary }} \times D
$$

The period $T P_{X}$ is the time during which the node of type $X$ keeps the same antenna configuration. The duration of a period, $T P$, is $D$ times longer than the time that an ordinary node keeps the same configuration, which means that $\mathrm{CH}$ nodes keep the same configuration during a complete cycle of an ordinary node.

$$
T P_{C H}=T C_{\text {Ordinary }}=T P_{\text {Ordinary }} \times D
$$

We deduce then that :

$$
T C_{C H}=T P_{\text {Ordinary }} \times D^{2}
$$

The use of two different period durations $T P_{C H}$ and $T P_{\text {Ordinary } y}$ prevents the hidden node cases, where two close nodes are not seeing each other because they are always directed in the same direction. During a given period, a node is either in listening, transmitting or sleeping mode [3].

\subsection{Clustering}

The clustering phase divides the nanonetwork into clusters with two levels: $\mathrm{CH}$ nodes and cluster member nodes. The clustering process aims to reduce the amount of transmitted information and interference risks. Every $\mathrm{CH}$ aggregates data sent by its member nodes, and relays it to neighboring $\mathrm{CHs}$ in order to reach the entire nanonetwork.

The $\mathrm{CH}$ selection is done in distributed, independent and random decision way. When a node becomes $\mathrm{CH}$, it will advertise (message $A D V)$ the neighboring ordinary nodes of its new rank during a complete cycle $T C_{C H}$.

In [11], authors stated that Terahertz bandwidth should be adapted according to the distance between communicating nodes. Three frequency windows are then proposed to carry-out shortrange, mean-range or long-range communications. The $\mathrm{CH}$ nodes select randomly a target range indicating the targeted distance from covered ordinary nodes. The $A D V$ message is then sent using frequency window associated with the selected range.

On the other hand, ordinary nodes work with a shorter cycle of duration $T C_{\text {ordinary }}$.

Ordinary nodes retrieve $A D V$ messages sent by neighboring $\mathrm{CHs}$, and decide to join one of them according to the quality of the received signal. Also, as indicated in figure 2, the $A D V$ message can be received by other $\mathrm{CHs}$ which allows to discover its $\mathrm{CH}$ neighbours (described in section 4.2.1).

Each member node informs the selected $\mathrm{CH}$ of its membership decision by a FOIN message. Subsequently, communications within a cluster are done using the TDMA technique and the selected frequency sub-band. For this, each $\mathrm{CH}$ establishes a TDMA schedule for its members to coordinate their communications. Communications between cluster heads are managed by the proposed distributed TDMA protocol and Forwarding Dominate Nodes algorithm (FDNs). FDN is described in section 4.2.2.

As indicated in figure 3 switching from one antenna direction to another one can take $D \times T P_{\text {ordinary }}$. So the ordinary nodes are ensured to receive an $(A D V s)$ message from all neighboring $\mathrm{CHs}$. When a $\mathrm{CH}$ takes an antenna direction $A D_{i}$, it has to send regularly $D$ times $A D V$ messages. The duration between sending $A D V s$ messages is the same as $T P_{\text {ordinary }}$.

4.2.1 $\mathrm{CH}$ neighborhood discovery. Upon the reception of an $\mathrm{ADV}$ message, the receiver $\mathrm{CH}_{j}$ adds the sender $\mathrm{CH}_{i}$ to its neighbouring $\mathrm{CHs}$ list named $\mathrm{N}_{\mathrm{CH}_{j}}$. This list includes all $\mathrm{CHs}$ directly linked to $\mathrm{CH}_{j}$ (accessible with just one hop). After receiving the $A D V s$ of different Cluster heads, each cluster head, $\mathrm{CH}_{j}$, sends its $\mathrm{N} \mathrm{CH}_{j}$ list to its neighboring cluster heads. At the end, each $\mathrm{CH}_{i}$ can apply algorithm 1 and chooses its Forwarding Dominate Nodes $\left(F D N_{C H_{i}}\right)$ among its neighbours. The $F D N_{C H_{i}}$ is described by the following formula:

$$
F D N_{C H_{i}}=\left\{C H_{j} \in \mathrm{N1}_{C_{C H}} / \mathrm{CH}_{j} \text { relays data received from } \mathrm{CH}_{i}\right\}
$$

The $\mathrm{FDN}_{\mathrm{CH}}$ list is then sent to the neighboring cluster heads, $\mathrm{CH}_{j} \in \mathrm{N} 1\left(\mathrm{CH}_{i}\right)$. Upon the reception of the $\mathrm{FDN}_{\mathrm{CH}_{i}}$ list, the neighboring cluster head, $\mathrm{CH}_{j}$ checks if it belongs to $\mathrm{FDN}_{\mathrm{CH}_{i}}$, if so, it adds the transmitter $\mathrm{CH}_{i}$ its selectors $\mathrm{S}_{\mathrm{CH}_{j}}$ list. For each cluster head, $\mathrm{CH}_{i}, \mathrm{~S}_{\mathrm{CH}_{i}}$ list includes neighboring cluster heads that have choosing $\mathrm{CH}_{i}$ as a $F D N$ node. $S_{C H_{j}}$ is defined by:

$$
S_{C H_{i}}=\left\{C H_{j} \in N 1_{C H_{i}} / C_{i} \in F D N_{C H_{j}}\right\}
$$




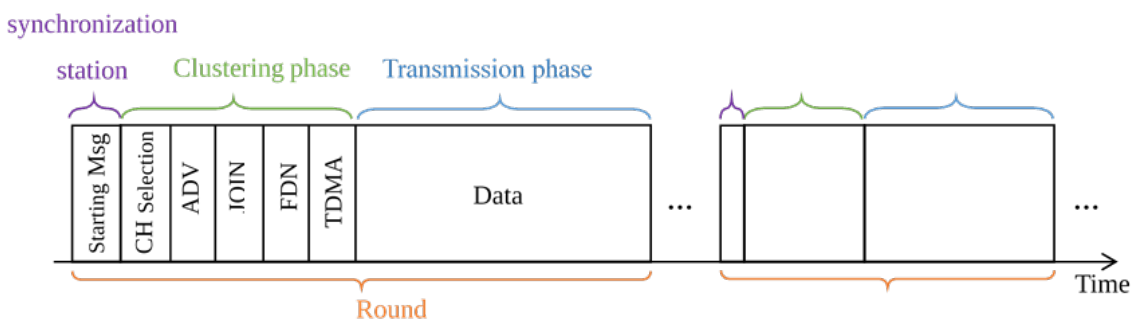

Figure 1: Clustering and communication phases

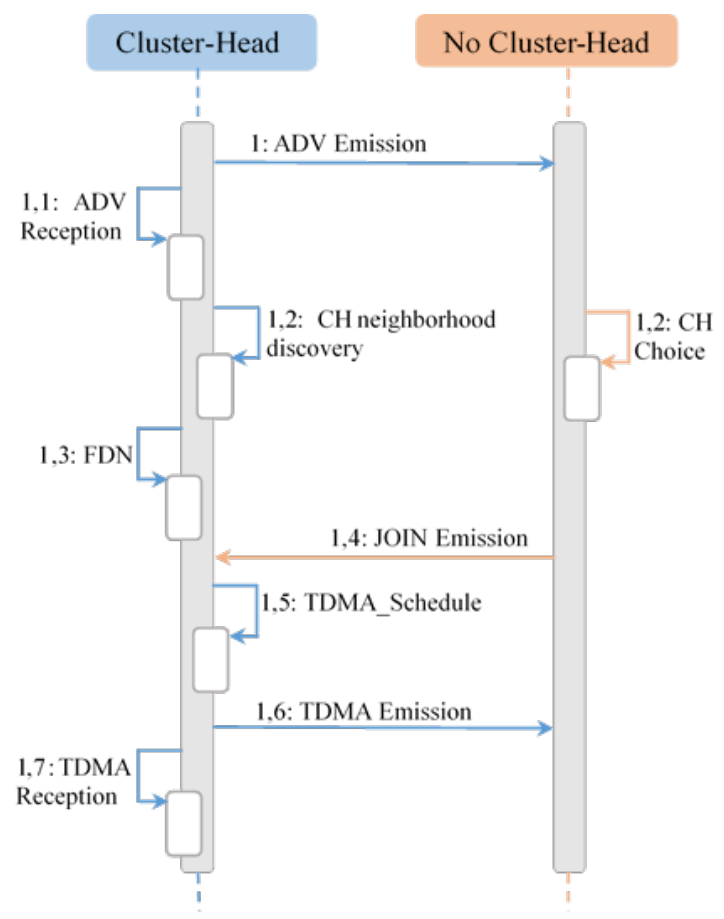

Figure 2: Sequence diagram of clustering phase

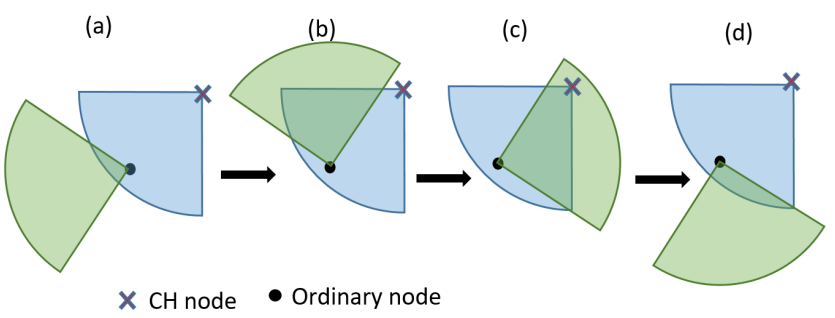

Figure 3: Switching Antennas in ADVs step. At (c) step, the ordinary node receives the ADV message.

We deduce that the sets FDN and $S$ are linked by the following expression:

$$
\mathrm{CH}_{i} \in S_{\mathrm{CH}_{j}} \Leftrightarrow \mathrm{CHj} \in \mathrm{FDN}_{C \mathrm{H}_{i}}
$$

Therefore, during transmission phase, each data packet transmitted by $\mathrm{CH}_{i}$ will be forwarded by only nodes $\mathrm{CH}_{j}$ such that $\mathrm{CH}_{i} \in \mathrm{S}_{\mathrm{CH}_{j}}$. For example in figure 4, data packet of $\mathrm{CH}$ node 4 will be forwarded by only $\mathrm{CH}$ nodes 7, 11 and 15 to finally reach all nodes.

4.2.2 Forward Dominate Nodes. The Forwarding Dominate Nodes is a distributed process that determine the subset of neighboring $\mathrm{CH}$ nodes that will act as relay nodes. The use of these subset of nodes enables to reduce the number of redundant retransmissions for broadcasting a packet over the nanonetwork. A packet is re-transmitted only by relay nodes contrary to a naive flood approach.

Let $\mathrm{CH}_{i}, \mathrm{N2}_{\mathrm{CH}_{i}}$ be a set of two hops $\mathrm{CHs}$ neighbors and $\mathrm{FDN}_{\mathrm{CH}}$ the relaying nodes set of $\mathrm{CH}_{i} . \mathrm{FDN}_{\mathrm{CH}}$ is initially empty.

- If a neighboring $\mathrm{CH}_{j} \in \mathrm{N} 1_{\mathrm{CH}_{i}}$ covers all the nodes of the set $\mathrm{N}_{2} \mathrm{CH}_{i}$, we define the node $\mathrm{CH}_{j}$ as member of $\mathrm{FDN}_{\mathrm{CH}}$ and we stop searching for other relay nodes for $\mathrm{CH}_{i}$.

- If there are nodes $\mathrm{CH}_{k} \in \mathrm{N}_{2} \mathrm{CH}_{i}$ which have one and only one link with a node $\mathrm{CH}_{j} \in \mathrm{N1}_{\mathrm{CH}_{i}}$, we add $\mathrm{CH}_{j}$ to the $F D N_{C H_{i}}$, and we remove from $\mathrm{N}_{2} \mathrm{CH}_{i}$ all the nodes connected to the added node $\mathrm{CH}_{j}\left(\mathrm{~N}_{\mathrm{CH}_{j}}\right)$.

- If a node $\mathrm{CH}_{k} \in \mathrm{N}^{2} \mathrm{CH}_{i}$ is linked to several $\mathrm{CH}$ nodes forming a subset $E \subset N 1_{C H_{i}}$, then the node $\mathrm{CH}_{j} \in E$ which covers a maximum number of nodes in $\mathrm{N}_{2} \mathrm{CH}_{i}$ is added to $\mathrm{FDN}_{\mathrm{CH}}$. If there are several nodes that cover the same number of nodes, the added $\mathrm{CH}$ is the one with the highest degree (number of neighbors $|N 1|)$. All nodes directly linked to the added node $\mathrm{CH}_{j}$ are then removed from $\mathrm{N}_{2} \mathrm{CH}_{i}$.

- The search for new relay nodes stops when the set $\mathrm{N}^{2} \mathrm{CH}_{i}$ becomes empty.

4.2.3 TDMA approaches. The TDMA-Schedule avoids collisions (Transmission channel access conflicts) between nodes and therefore minimizes nodes power consumption by reducing the retransmission effort. In addition, it allows node to turn off its communication interface outside its reserved time slot that is used to transmit its sensed/computed data.

TDMA is a temporal multiplexing technique where each served node uses a particular time slot. In our approach the TDMA scheme is used at two levels: Distributed inter cluster and centralized intra cluster scheduling.

4.2.4 Intra Cluster TDMA. After the construction of clusters, each $\mathrm{CH}$ builds the TDMA-Schedule and informs its members by 


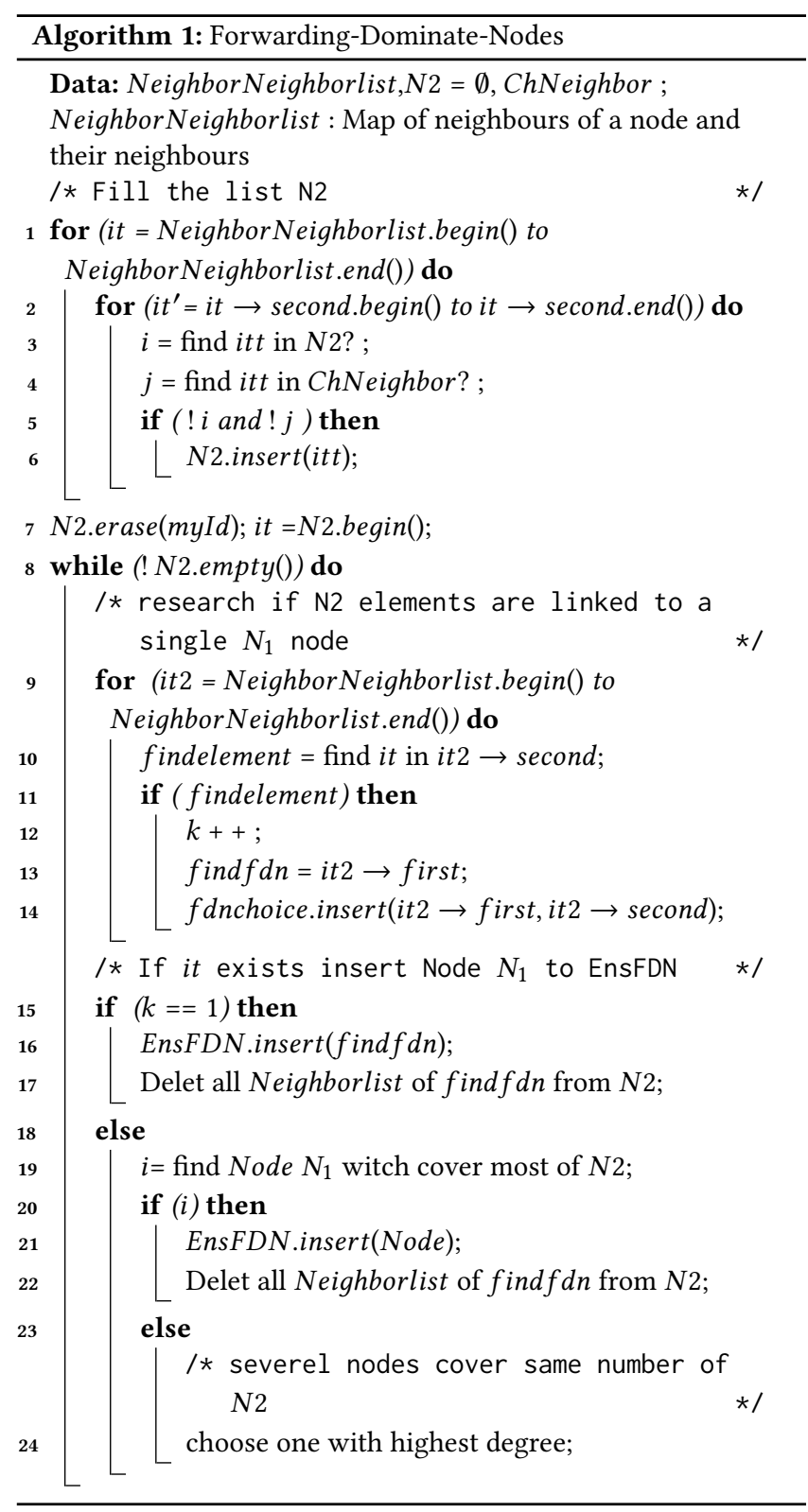

their rank in this TDMA-Schedule. The rank of member node indicates the time slot during which the node sends its sensed/computed data to its own $\mathrm{CH}$. An ordinary node, $n$, calculates its time slot $T S_{n}$ as follows:

$$
T S_{n}=\operatorname{rank}_{n} * T s
$$

Where $T s$ is the slot duration.

4.2.5 Inter Cluster TDMA. Each cluster head runs the algorithm 2 to fix the time slots for communicating with its direct neighboring CHs: $\mathrm{N}_{\mathrm{CH}}$. First, the $\mathrm{CHs}$ with the smallest identity number among their direct neighbors begin the computation of their TDMASchedule and transmit it to their neighbors (See steps 1.5 and 1.6 in Figure 2). Once the TDMA-Schedules of all the neighbors with

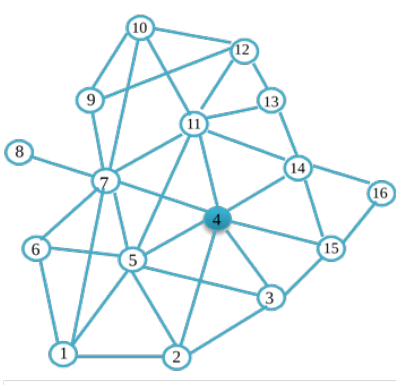

(a) Flood the network requires as many transmissions as the number of nodes

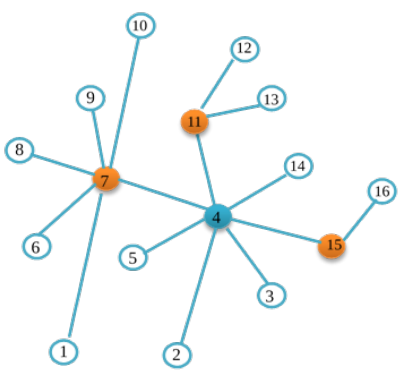

(b) Flood the entire network with 4 transmissions using FDN
Figure 4: Flood diffusion (left) and optimized diffusion (right)

smaller identities are received (step 1.7 in Figure 2), the $\mathrm{CH}$ schedules its own TDMA-Schedule according to the schedules already done.

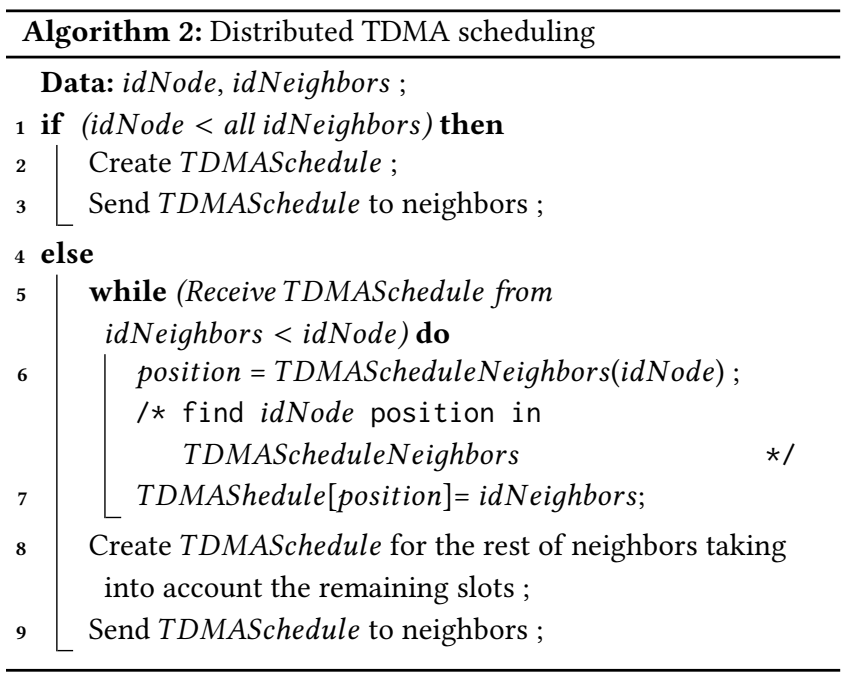

The figure 5 shows an example of time slots planning over $13 \mathrm{CHs}$. At the beginning, each $\mathrm{CH}$ checks if it has the smallest identifier regarding to its neighbors. $\mathrm{CH} 1, \mathrm{CH} 2$ and $\mathrm{CH} 8$ start the construction of their TDMA-Schedule in first. When they send their tables to their respective neighbors, the other $\mathrm{CHs}$ establish in turn their own TDMA-Schedules. The $\mathrm{CH} 3, \mathrm{CH}, \mathrm{CH} 7$ and $\mathrm{CH} 10$ have been released by their neighbor $\mathrm{CH} 1$ while $\mathrm{CH} 12$ has been released by $\mathrm{CH} 1$ and $\mathrm{CH} 8$. This process is repeated until all $\mathrm{CHs}$ obtain a TDMA-Schedule.

After the clustering phase, the $\mathrm{CH}$ nodes return to the cycle of $T C_{\text {ordinary }}$, while the ordinary nodes keeps the same antenna configuration all the time (the direction corresponding to the selected $\mathrm{CH})$. Thus, the transmission phase starts. Each node transmits its sensed/camputed data. These data received by the covering $\mathrm{CH}$ are then aggregated and transmitted to other neighboring $\mathrm{CHs}$ which in their turn send them to their $\mathrm{CH}$ neighbors and theirs members according to the FDN lists. 

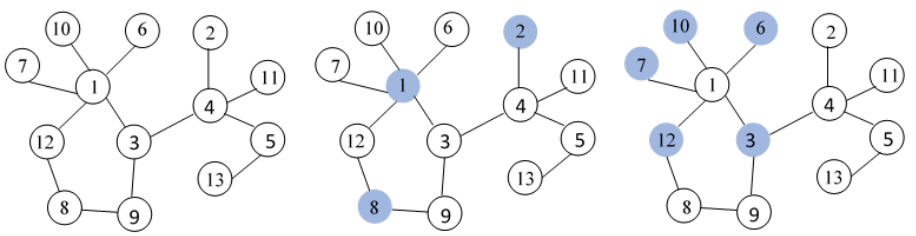

$$
\begin{aligned}
& \text { 1: }\{3,6,7,10,12\} \\
& \mathbf{2}:\{4\} \\
& \mathbf{8}:\{9,12\}
\end{aligned}
$$

$3:\{1,4,9\}$

$6:\{/, 1\}$

$7:\{/, /, 1\}$

$10:\{/, /, /, 1\}$
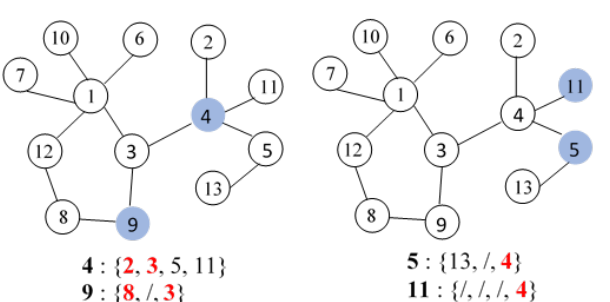

$12:\{/, 8, /, /, 1\}$

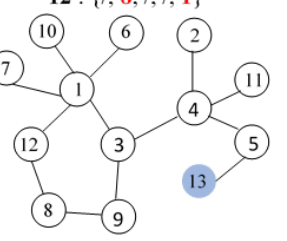

$4:\{2,3,5,11\}$

$11:\{/, /, /, 4\}$

$13:\{/, /, /, 5\}$

Figure 5: Distributed TDMA inter cluster scheduling

\section{EXPERIMENTAL TESTS}

To assess the performance of proposed traffic regulation protocol, simulations was done using BitSimulator platform [9]. BitSimulator presents a good testing framework, because it provides a high level of accuracy (at a symbol level) and high calculation performances (simulation of dense terahertz nanonetworks).

The high density of a nanonetwork necessarily generates huge messages traffic. The lack of regulation of the traffic will negatively affect the overall behavior of the nanonetwork. As well, to consider the contribution of our clustering algorithm dedicated to the Directional Antennas Nanonetwork, we used several test scenarios including the number of nodes and the number of directional antennas. This enabled to assess the impact of clustering and TDMA schedules on the performance of the nanonetwork in terms of generated interference. So we compared the number of messages generated by our proposal with the flooding approach.

Figure 6 shows the results obtained on several scenarios with different number of nodes deployed over an area of $6 \mathrm{~mm} \times 6 \mathrm{~mm}$ with a communication range of $0.5 \mathrm{~mm}$. For each scenario, we give the number of sent messages in the flooding and in our clustering approach. The curves show that the performance of our proposal outperforms that of the flooding. Our proposal presents a significant gain of about $15 \%$ of the total messages used by flooding approach. This result is interesting knowing that in a nanonetwork the very high nodes density is the prime source of messages traffic perturbation.

The number of messages generated by the broadcasting of a data over the network with the flooding approach can be computed as follow:

$$
N b M s g=\sum_{i=1}^{C} N 1_{n_{i}}
$$

Where $C$ is the total number of nodes and $N 1_{n_{i}}$ is the number of neighboring nodes of the node, $n_{i}$.
In the clustering approach, the maximum number of messages generated by data broadcasting from an ordinary node, $n$, to all the nodes is given by the following recursive formula:

$$
\begin{gathered}
\operatorname{NbMsg}(n)=1+\alpha_{C h_{i}}+\operatorname{NbMsg}\left(C h_{i} / n \text { is covered by } C h_{i}\right) \\
N b M s g\left(C h_{i}\right)=\sum_{C h_{j} \in N 1_{C h_{i}}}\left\{\begin{array}{cc}
N b M s g\left(C h_{j}\right) & \text { if } C h_{j} \in F D N_{C h_{i}} \\
D & \text { else }
\end{array}\right.
\end{gathered}
$$

where $\alpha_{C h_{i}}=D+N 1_{C h_{i}}$ and $D$ is the number of antenna directions.

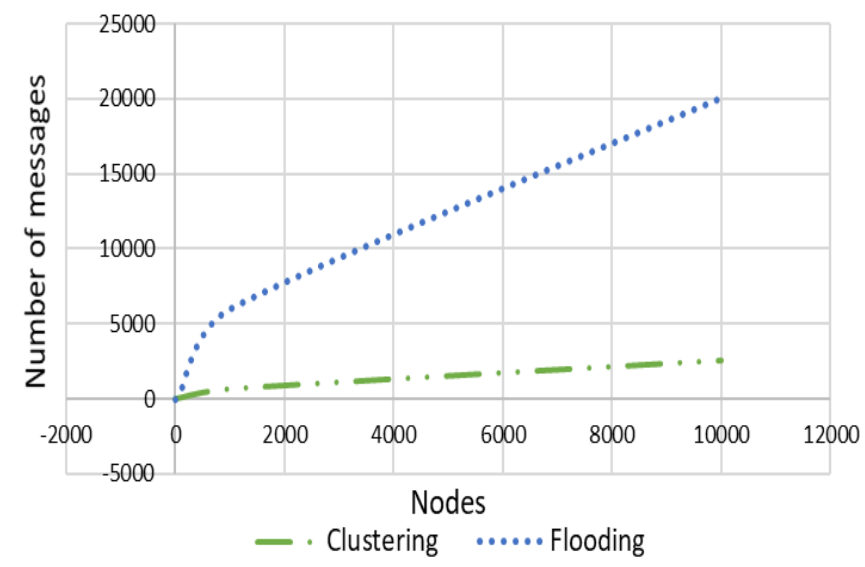

Figure 6: Clustering vs Flooding regarding number of messages generated. Tests are made with different network densities.

We now study the impact of cluster heads number on the performances of the clustering method. Too much or too little cluster heads number impacts on the nano nodes lifetime. so it has a great 
significance to select the optimal cluster heads number for the nanonetwork. The higher the number of $\mathrm{CHs}$, the more energy will be consumed. This is due to the increase of communications number between cluster heads (see eq. 9). Futhermore, the number of clusters is inversely proportional to the clusters size. Thus, the latency driven by TDMA protocol is directly proportional to the size of clusters.

Figure 7 shows two curves obtained from simulation over a nanonetwork of 1000 nodes. The first curve indicates the variation of exchanged message number according to the number of $\mathrm{CHs}$ (given as percentage). We note that the number of messages increases with the percentage of $\mathrm{CHs}$ until a given threshold. When the percentage of $\mathrm{CHs}$ exceeds $75 \%$, the number of messages decreases, because messages between $\mathrm{CHs}$ and their respective members disappear.

Furthermore, when the number of $\mathrm{CHs}$ is small, the number of nodes receiving the data is small too (see the dotted curve in figure 7). For example, with $2 \%$ of $\mathrm{CHs}$ only $40 \%$ of the nodes received the data. This is due to the fact that, when the $\mathrm{CHs}$ percentage is low, some individual or groups of nodes become isolated. Beyond 10\% of $\mathrm{CHs}$, we note that the data reaches the entire network.

With regard to collisions, intra cluster collisions are impossible. Therefore, under the condition that data generated by each node are not voluminous and relatively less frequent while the number of simultaneous messages generated by all nodes is high, the TDMA method performs better than random access approach or CDMA/CA method.

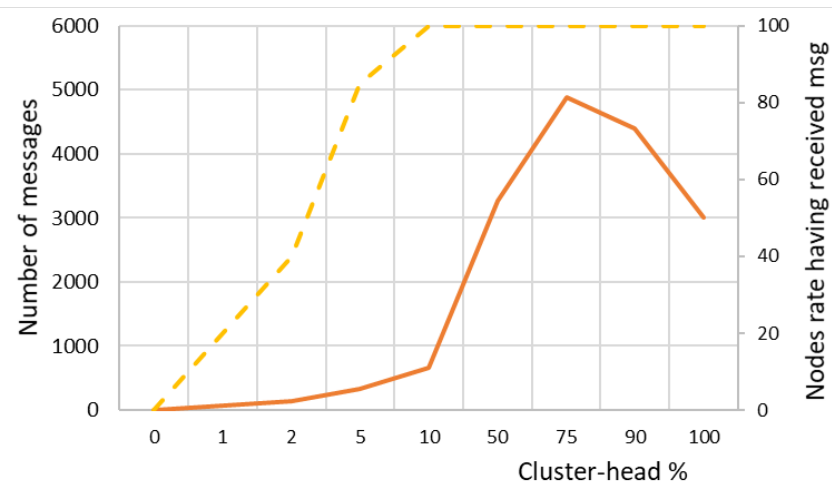

Number of messages

- Nodes rate having received msg

Figure 7: Optimal $\mathrm{CH}$ rate for covering the entire nanonetwork

\section{CONCLUSION}

Efficient coordination of communications in WNN with very high nodes density allows to significantly reduce the costly conflicting channel accesses. Consequently, collisions are minimized and node energy consumption too. To this end, our proposal uses directional antennas equipping nodes to better steering signals for accurate nodes discovery. This allows contributing to achieve clusters formation and controlling multilevel node communications. The first level concerns communications inside clusters with use of TDMA approach while the second level is dedicated to inter cluster communications with help of forwarding dominate nodes and uses a new proposal distributed TDMA scheme. This multilevel approach allows regulating the high number of data generated by the nanonetwork nodes by scheduling the transmissions and optimizing the data routing.

The proposed approach takes into account the particularities of the Terahertz band. Every cluster head corresponds to a given frequency windows adapted to the distance between the cluster head and the member nodes. In this way, the undesirable intercluster interference is also reduced.

As future work, we envision to improve the proposal by reducing the number of time slots in the CH's TDMA schedule in such way to keep the same transmission slot for all neighboring $\mathrm{CHs}$ present at the same direction. The objective is the reduction of the TDMA schedule size in order to give $\mathrm{CHs}$ a rest time for vital recharging their batteries.

\section{REFERENCES}

[1] Ji Hyoung Ahn and Tae-Jin Lee. 2014. Multipoint relay selection for robust broadcast in ad hoc networks. Ad Hoc Networks 17 (2014), 82-97.

[2] Ian F Akyildiz and Josep Miquel Jornet. 2010. Electromagnetic wireless nanosensor networks. Nano Communication Networks 1, 1 (2010), 3-19.

[3] Lina Aliouat, Hakim Mabed, and Julien Bourgeois. 2018. 2.5 Layer Protocol for Traffic Regulation in Ultra-Dense Nanonetwork. In 2018 IEEE 17th International Symposium on Network Computing and Applications (NCA). IEEE, 1-5.

[4] Baris Atakan, Ozgur B Akan, and Sasitharan Balasubramaniam. 2012. Body area nanonetworks with molecular communications in nanomedicine. IEEE Communications Magazine 50, 1 (2012).

[5] Nicolas Boillot, Dominique Dhoutaut, and Julien Bourgeois. 2015. Large scale mems robots cooperative map building based on realistic simulation of nanowireless communications. Nano Communication Networks 6, 2 (2015), 51-73.

[6] Y. Fu I. M. Chio M. DeLisio Chiao, J. and L. Y. Lin. 1999. MEMS reconfigurable vee antenna. IEEE MTT-S International Microwave Symposium Digest 4, 1515-1518.

[7] Diego Correas Serrano and J S. Gomez-Diaz. 2017. Graphene-based Antennas for Terahertz Systems: A Review. (04 2017), 1-26.

[8] Ruyuan Deng, Fan Yang, Shenheng Xu, and Paola Pirinoli. 2014. Terahertz Reflectarray Antennas: An overview of the State-of-the-art Technology. International Conference on Electromagnetics in Advanced Applications, 667-670.

[9] Dominique Dhoutaut, Thierry Arrabal, and Eugen Dedu. 2018. BitSimulator, an electromagnetic nanonetworks simulator. In 5th ACM/IEEE International Conference on Nanoscale Computing and Communication (NanoCom). ACM/IEEE, Reykjavik, Iceland, 1-6.

[10] Bo Han and Weijia Jia. 2007. Clustering wireless ad hoc networks with weakly connected dominating set. f. Parallel and Distrib. Comput. 67, 6 (2007), 727-737.

[11] Chong Han and Ian F Akyildiz. 2014. Distance-aware multi-carrier (DAMC) modulation in terahertz band communication. In Communications (ICC), 2014 IEEE International Conference on. IEEE, 5461-5467.

[12] Josep Miquel Jornet and Ian F Akyildiz. 2010. Graphene-based nano-antennas for electromagnetic nanocommunications in the terahertz band. In Proceedings of the Fourth European Conference on Antennas and Propagation. IEEE, 1-5.

[13] Josep Miquel Jornet and Ian F Akyildiz. 2014. Femtosecond-long pulse-based modulation for terahertz band communication in nanonetworks. IEEE Transactions on Communications 62, 5 (2014), 1742-1754.

[14] Josep Miquel Jornet, Joan Capdevila Pujol, and Josep Solé Pareta. 2012. Phlame: A physical layer aware mac protocol for electromagnetic nanonetworks in the terahertz band. Nano Communication Networks 3, 1 (2012), 74-81.

[15] Bérengère Lebental, Boutheina Ghaddab, and Fulvio Michelis. 2015. Nanosensors for Embedded Monitoring of Construction Materials: The "2D Conformable" Route. In Nanotechnology in Construction. Springer, 383-388.

[16] Cen Lin and Geoffrey Ye Li. 2015. Adaptive Beamforming With Resource Allocation for Distance-Aware Multi-User Indoor Terahertz Communications. IEEE Trans. Communications 63, 8 (2015), 2985-2995.

[17] Xuxun Liu. 2012. A survey on clustering routing protocols in wireless sensor networks. sensors 12, 8 (2012), 11113-11153.

[18] Hakim Mabed. 2017. Enhanced spread in time on-off keying technique for dense Terahertz nanonetworks. In Computers and Communications (ISCC), 2017 IEEE Symposium on. IEEE, 710-716.

[19] Vitaly Petrov, Alexander Pyattaev, Dmitri Moltchanov, and Yevgeni Koucheryavy. 2016. Terahertz band communications: Applications, research challenges, and standardization activities. 183-190. https://doi.org/10.1109/ICUMT.2016.7765354 
[20] Giuseppe Piro, Luigi Alfredo Grieco, Gennaro Boggia, and Pietro Camarda. 2013. Nano-Sim: simulating electromagnetic-based nanonetworks in the network simulator 3. In Proc. of the 6th Intl. ICST Conf. on Simulation Tools and Techniques. ICST, 203-210.

[21] Lakshmi Ramachandran, Manika Kapoor, Abhinanda Sarkar, and Alok Aggarwal 2000. Clustering Algorithms for Wireless Ad Hoc Networks. In Proceedings of the 4th International Workshop on Discrete Algorithms and Methods for Mobile Computing and Communications (DIALM '00). ACM, 54-63.

[22] Negar Rikhtegar, Manijeh Keshtgari, and Zahra Ronaghi. 2017. EEWNSN: Energy Efficient Wireless Nano Sensor Network MAC Protocol for Communications in the Terahertz Band. Wireless Personal Communications 97, 1 (2017), 521-537.

[23] Aggeliki Sgora, Dimitrios J Vergados, and Dimitrios D Vergados. 2015. A survey of TDMA scheduling schemes in wireless multihop networks. ACM Computing Surveys (CSUR) 47, 3 (2015), 53.

[24] V Srikanth, Sindhu Chaluvadi, et al. 2012. Energy efficient, scalable and reliable MAC protocol for electromagnetic communication among nano devices. International Journal of Distributed and Parallel Systems 3, 1 (2012), 249.

[25] Iyemeh Uchendu and J. R. Kelly. 2016. Survey of Beam Steering Techniques Available for Millimeter Wave Applications. Progress In Electromagnetics Research B 68 (2016), 35-54.

[26] Pu Wang, Josep Miquel Jornet, MG Abbas Malik, Nadine Akkari, and Ian F Akyildiz. 2013. Energy and spectrum-aware MAC protocol for perpetual wireless nanosensor networks in the Terahertz Band. Ad Hoc Networks 11, 8 (2013), $2541-2555$ 